\title{
Analysis of Antimicrobial Resistance of Microbiota Associated with Respiratory Diseases of Pigs
}

\author{
Ilya Matrenov*, Olga Larionova, and Yaroslav Drevko \\ Federal State-Owned Publicly-Funded Institution of Higher Education Saratov State Agrarian \\ University named after N.I. Vavilov, Department of Microbiology, Biotechnology and Chemistry, \\ Saratov, 410012, Russia, 1 Teatralnaya pl.
}

\begin{abstract}
Successful treatment of veterinary infectious diseases in the context of widespread use of antibiotic therapy depends on the effectiveness of the drugs used to contain or destroy the etiological agent. It is generally accepted that the resistance of microorganisms to antimicrobial agents is variable, including due to the horizontal movement of resistance with the help of mobile genetic elements. Antimicrobial susceptibility pattern analysis is an important component of the diagnosis and treatment of veterinary diseases. The article describes a study that includes isolation and identification of microorganisms from samples taken from pathological material of pigs and the analysis of sensitivity to eleven antimicrobial drugs by the disk-diffusion method.
\end{abstract}

\section{Introduction}

Successful treatment of veterinary infectious diseases in the context of widespread use of antibiotic therapy depends on the effectiveness of the drugs used to contain or destroy the etiological agent [1-4]. It is generally accepted that the resistance of microorganisms to antimicrobial agents is variable, including due to the horizontal movement of resistance with the help of mobile genetic elements [5-7].

In order to monitor the sensitivity of microbiota to antimicrobial substances, samples of pathological material from pig corpses from pig breeding complexes in Russia were taken from March to May 2021. The species identification of the isolated microorganisms was determined and the parameters of sensitivity to antimicrobial substances, which are widely presented on the market in the composition of medicines for veterinary use, were determined.

\section{Methods}

In laboratory conditions, 14 washes were analyzed from pathological material from pig corpses. Washes were delivered within 24 hours in a heat-insulating box equipped with

* Corresponding author: matrenov.is@yandex.ru 
cold accumulators at a temperature not exceeding $8{ }^{\circ} \mathrm{C}$, which was confirmed by the readings of an electronic thermometer with the function of periodically recording readings. The Cary-Blair medium was used as a transport medium. The studies were carried out in accordance with the CLSI recommendations [8] by the disk-diffusion method. A set of antibiotics of the following composition was used: amoxicillin (AMC), amoxicillin + clavulanic acid (AMK), azitronite (AZT), doxycycline (DXC), colistin (COL), levofloxacin (LVC), tiamulin (TML), tilmicosin (TMS), florfenicol (FLF), ceftiofur (CFF), enrofloxacin (EFC). The washing sampling sites were determined by clinical signs similar to the manifestations of pig pleuropneumonia and hemophillosis, as well as the pathological picture of the autopsy objects. The isolated isolates were identified using API biochemical tests developed and manufactured by BioMérieux. The API test results were interpreted using the APIWeb service. The experimental design included 6 parallel measurements of the sensitivity of one strain to one antimicrobial substance $(n=6)$. The results were statistically processed according to standard procedures using Microsoft Excel 2016 (Microsoft Corp. USA) and the statistical data analysis package StatPlus 2009 Professional 5.8.4 for Windows (StatSoftInc., USA) [9]. The experiment used a control strain of the museum culture E. coli ATCC 25922.

\section{Results}

The study results are shown in Table 1.

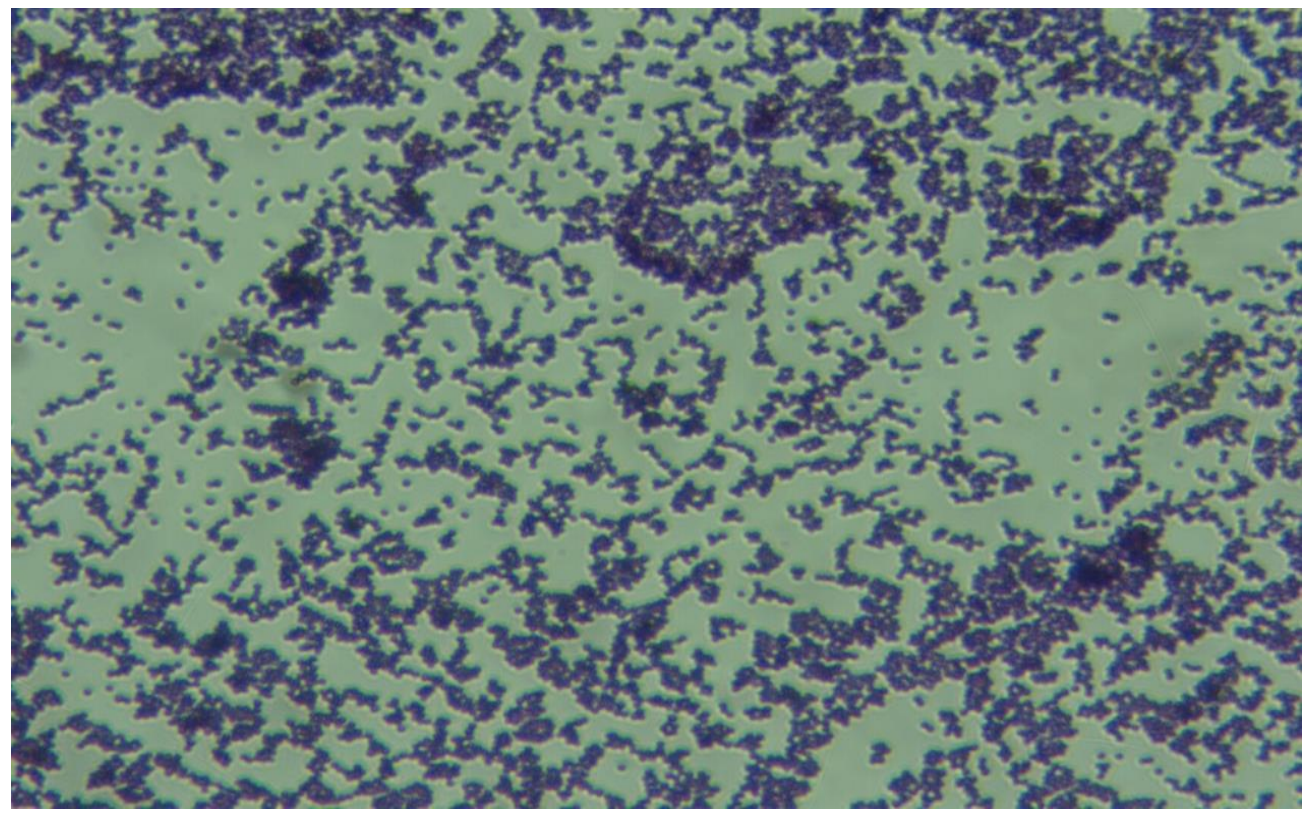

Figure 1. Isolate of Streptococcus suis (No. 1, Table 1), $\times 1000$ magnification. 


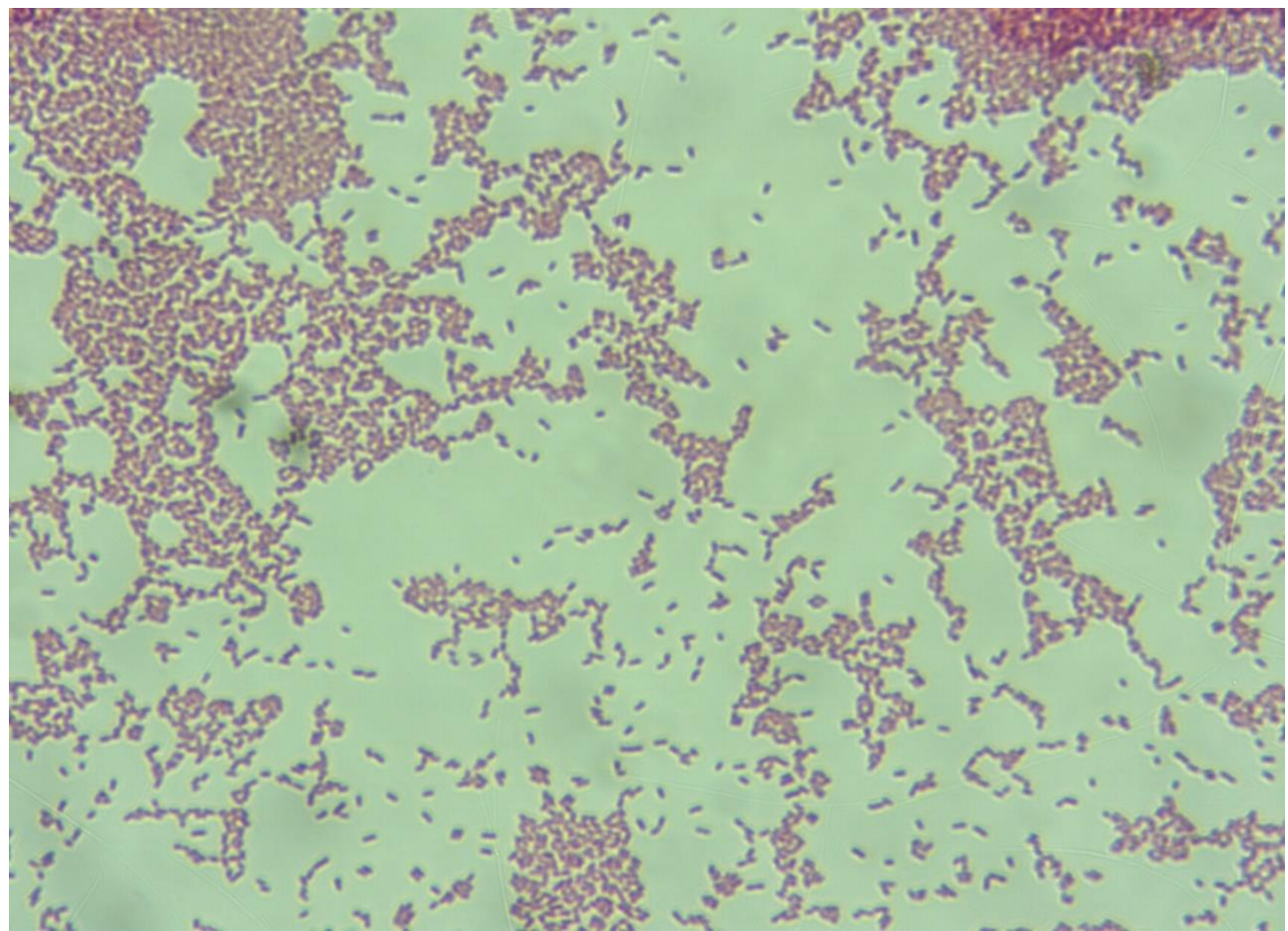

Figure 2. Proteus mirabilis isolate (No. 3, Table 1), $\times 1000$ magnification.

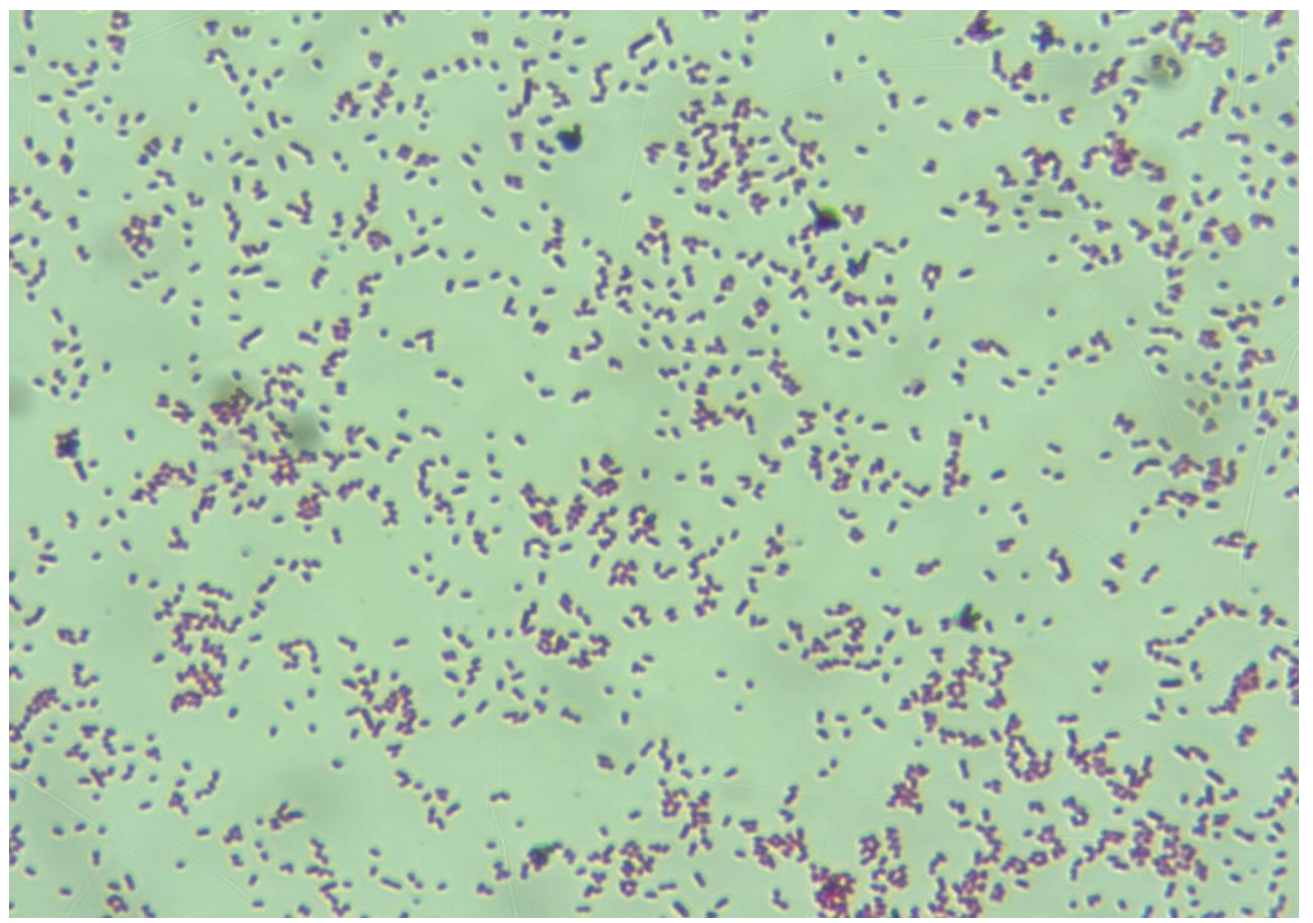

Figere 3. Morganella morganii isolate (No. 21, Table 1), $\times 1000$ magnification. 
Table 1. Study Results.

\begin{tabular}{|c|c|c|c|c|c|c|c|}
\hline \multirow{2}{*}{\multicolumn{2}{|c|}{$\begin{array}{c}\text { Microorganism, place } \\
\text { of washing }\end{array}$}} & \multicolumn{6}{|c|}{ Inhibition zones, $\mathbf{m m}$} \\
\hline & & AMC & AMK & $\mathbf{A Z T}$ & DXC & COL & LVC \\
\hline 1 & $\begin{array}{l}\text { Streptococcus suis } \\
\text { (synovial fluid) }\end{array}$ & $30 \pm 1.4$ & $\begin{array}{c}28.3 \pm \\
1.7\end{array}$ & $\begin{array}{c}18.2 \pm 1 \\
, .6\end{array}$ & $19.5 \pm 0.8$ & 0 & $24 \pm 1.3$ \\
\hline 2 & $\begin{array}{l}\text { E.coli (exudate, } \\
\text { lungs) }\end{array}$ & $9.8 \pm 0.9$ & 0 & $\begin{array}{c}28.7 \pm 2 \\
.1\end{array}$ & $15 \pm 1$ & $17.3 \pm 1.4$ & $\begin{array}{c}32.8 \pm 1 \\
7\end{array}$ \\
\hline 3 & $\begin{array}{l}\text { Proteus mirabilis } \\
\text { (exudate, lungs) }\end{array}$ & $9.3 \pm 1.1$ & 0 & $\begin{array}{c}11.7 \pm 0 \\
.8\end{array}$ & $10.3 \pm 1.5$ & 0 & $\begin{array}{c}22.7 \pm 1 \\
7\end{array}$ \\
\hline 4 & E. c oli (liver) & $8.2 \pm 0.8$ & 0 & $\begin{array}{c}14.3 \pm 0 \\
.4\end{array}$ & $9.8 \pm 0.6$ & $14.3 \pm 0.8$ & $\begin{array}{c}26.8 \pm 1 \\
9\end{array}$ \\
\hline 5 & $\begin{array}{l}\text { Myroides phaeus } \\
\text { (liver) }\end{array}$ & 0 & $\begin{array}{c}28.3 \pm \\
1.1\end{array}$ & $\begin{array}{c}28.2 \pm 1 \\
.9\end{array}$ & $15.2 \pm 1.1$ & $10.2 \pm 0.6$ & 0 \\
\hline 6 & $\begin{array}{l}\text { Streptococcus } \\
\text { canis } \\
\text { (pericardium) }\end{array}$ & $\begin{array}{c}34.2 \pm 1 \\
1\end{array}$ & $\begin{array}{c}30 \pm 1 \\
1\end{array}$ & $\begin{array}{c}17.2 \pm 1 \\
.1\end{array}$ & $12.2 \pm 1.1$ & $14.1 \pm 0.7$ & $\begin{array}{c}24.3 \pm 1 \\
1\end{array}$ \\
\hline 7 & $\begin{array}{l}\text { E. c oli } \\
\text { (pericardium) }\end{array}$ & $\begin{array}{c}21.2 \pm 1 \\
1\end{array}$ & $\begin{array}{c}20.7 \pm \\
1.2\end{array}$ & $\begin{array}{c}23.2 \pm 1 \\
.3\end{array}$ & $19.2 \pm 0.9$ & $14.6 \pm 0.7$ & $\begin{array}{c}30.8 \pm 1 \\
6\end{array}$ \\
\hline 8 & $\begin{array}{l}\text { E. c oli (exudate, } \\
\text { lungs) }\end{array}$ & $\begin{array}{c}10.8 \pm 0 \\
6\end{array}$ & $\begin{array}{c}18 \pm 0 \\
7\end{array}$ & $\begin{array}{c}14.2 \pm 0 \\
.6\end{array}$ & $13.1 \pm 0.6$ & $12.8 \pm 0.8$ & $\begin{array}{c}25.2 \pm 1 \\
5\end{array}$ \\
\hline 9 & $\begin{array}{l}\text { Proteus mirabilis } \\
\text { (exudate, lungs) }\end{array}$ & 0 & $\begin{array}{c}9.2 \pm 0 \\
6\end{array}$ & $\begin{array}{c}12.2 \pm 0 \\
.6\end{array}$ & $8.8 \pm 0.6$ & 0 & $\begin{array}{c}38.2 \pm 1 \\
1\end{array}$ \\
\hline 10 & $\begin{array}{l}\text { Klebsiella } \\
\text { pneumonia } \\
\text { (exudate, lungs) }\end{array}$ & $\begin{array}{c}11.2 \pm 0 \\
6\end{array}$ & $\begin{array}{c}20 \pm 1 \\
3\end{array}$ & $\begin{array}{c}22.7 \pm 1 \\
.5\end{array}$ & $16.2 \pm 0.6$ & $14.2 \pm 0.6$ & $\begin{array}{c}27.3 \pm 1 \\
2\end{array}$ \\
\hline 11 & $\begin{array}{l}\text { Providencia } \\
\text { rettgeri (exudate, } \\
\text { lungs) }\end{array}$ & 0 & 0 & $\begin{array}{c}13.8 \pm 0 \\
.8\end{array}$ & 0 & 0 & $\begin{array}{c}18.2 \pm 0 \\
6\end{array}$ \\
\hline 12 & $\begin{array}{l}\text { E. c oli } \\
\text { (pericardium) }\end{array}$ & 0 & $\begin{array}{c}13 \pm 0 \\
5\end{array}$ & $\begin{array}{c}17.5 \pm 1 \\
.2\end{array}$ & $11.2 \pm 0.8$ & 0 & $\begin{array}{c}17.8 \pm 0 \\
6\end{array}$ \\
\hline 13 & $\begin{array}{l}\text { Myroides } \\
\text { odoratus } \\
\text { (pericardium) }\end{array}$ & 0 & 0 & $\begin{array}{c}19.3 \pm 0 \\
.8\end{array}$ & $10.8 \pm 0.6$ & 0 & $14 \pm 0.5$ \\
\hline 14 & $\begin{array}{l}\text { E.coli (exudate, } \\
\text { lungs) }\end{array}$ & $10 \pm 0.7$ & $\begin{array}{c}9.2 \pm 0 \\
6\end{array}$ & $\begin{array}{c}14.3 \pm 0 \\
.8\end{array}$ & $15.1 \pm 0.7$ & $13.9 \pm 0.8$ & $\begin{array}{c}24.8 \pm 0 \\
6\end{array}$ \\
\hline 15 & $\begin{array}{l}\text { Proteus mirabilis } \\
\text { (exudate, lungs) }\end{array}$ & 0 & $\begin{array}{c}9.8 \pm 0 \\
8\end{array}$ & $\begin{array}{c}9.9 \pm 0 \\
7\end{array}$ & $9.3 \pm 0.7$ & 0 & $\begin{array}{c}35.8 \pm 0 \\
6\end{array}$ \\
\hline 16 & $\begin{array}{l}\text { Vagococcus } \\
\text { fluvialis (exudate, } \\
\text { lungs) }\end{array}$ & $\begin{array}{c}20.3 \pm 1 \\
2\end{array}$ & $\begin{array}{c}21.3 \pm \\
1.3\end{array}$ & 0 & $14.2 \pm 0.8$ & 0 & $\begin{array}{c}14.2 \pm 0 \\
8\end{array}$ \\
\hline 17 & E. c oli (exudate, & $21 \pm 1.3$ & $\begin{array}{c}20.8 \pm \\
1.1 \\
\end{array}$ & $27 \pm 0.5$ & $10.2 \pm 0.8$ & $13 \pm 0.5$ & $30 \pm 0.9$ \\
\hline
\end{tabular}




\begin{tabular}{|c|c|c|c|c|c|c|c|}
\hline \multirow{2}{*}{\multicolumn{2}{|c|}{$\begin{array}{l}\text { Microorganism, place } \\
\text { of washing }\end{array}$}} & \multicolumn{6}{|c|}{ Inhibition zones, mm } \\
\hline & & AMC & AMK & AZT & DXC & COL & LVC \\
\hline \multicolumn{8}{|c|}{ lungs) } \\
\hline 18 & $\begin{array}{l}\text { Proteus mirabilis } \\
\text { (exudate, lungs) }\end{array}$ & $\begin{array}{l}11.8 \pm 0 . \\
\quad 6\end{array}$ & $\begin{array}{c}10.2 \pm \\
0.6\end{array}$ & $\begin{array}{c}8.8 \pm 0 \\
8\end{array}$ & $9 \pm 0.9$ & 0 & $26 \pm 1.1$ \\
\hline 19 & $\begin{array}{l}\text { Aeromonas } \\
\text { hydrophila } \\
\text { (exudate, lungs) }\end{array}$ & 0 & 0 & $\begin{array}{c}14.2 \pm 0 \\
.8\end{array}$ & $9.2 \pm 1$ & 0 & $\begin{array}{c}15.3 \pm 1 \\
1\end{array}$ \\
\hline 20 & $\begin{array}{l}\text { E. c oli (synovial } \\
\text { fluid) }\end{array}$ & $18 \pm 0.9$ & $\begin{array}{c}20.3 \pm \\
1.1\end{array}$ & $\begin{array}{c}24.2 \pm 1 \\
.2\end{array}$ & $9.8 \pm 0.6$ & $14 \pm 0.7$ & $\begin{array}{l}27.8 \pm 0 . \\
8\end{array}$ \\
\hline 21 & $\begin{array}{l}\text { Morganella } \\
\text { morganii } \\
\text { (synovial fluid) }\end{array}$ & 0 & 0 & 0 & 0 & 0 & $22 \pm 0.9$ \\
\hline 22 & $\begin{array}{l}\text { E. c oli (exudate, } \\
\text { lungs) }\end{array}$ & $16 \pm 0.9$ & $\begin{array}{c}20.3 \pm \\
1.1\end{array}$ & $25 \pm 1.1$ & $9 \pm 0.5$ & $14 \pm 0.5$ & $\begin{array}{c}26.8 \pm 1 \\
1\end{array}$ \\
\hline 23 & $\begin{array}{l}\text { Proteus mirabilis } \\
\text { (exudate, lungs) }\end{array}$ & $\begin{array}{l}17.2 \pm 0 \\
\quad 8\end{array}$ & $\begin{array}{c}22 \pm 1 \\
1\end{array}$ & $\begin{array}{l}12.8 \pm 0 \\
.6\end{array}$ & $8.8 \pm 0.6$ & 0 & $\begin{array}{c}25.3 \pm 1 \\
1\end{array}$ \\
\hline 24 & $\begin{array}{l}\text { Providencia } \\
\text { rettgeri (exudate, } \\
\text { lungs) }\end{array}$ & $\begin{array}{c}23.7 \pm 1 \\
1\end{array}$ & $\begin{array}{c}26.2 \pm \\
1.1\end{array}$ & $\begin{array}{l}12.8 \pm 0 \\
.8\end{array}$ & 0 & 0 & $30 \pm 1.1$ \\
\hline 25 & $\begin{array}{l}\text { Aeromonas } \\
\text { hydrophila } \\
\text { (exudate, lungs) }\end{array}$ & 0 & 0 & $11 \pm 0.5$ & 0 & 0 & $\begin{array}{l}17.2 \pm 0 . \\
6\end{array}$ \\
\hline 26 & $\begin{array}{l}\text { Control E. coli } \\
\text { ATCC } 25922\end{array}$ & $\begin{array}{l}26.2 \pm 1 \\
3\end{array}$ & $\begin{array}{c}27.8 \pm \\
1.5\end{array}$ & $36 \pm 0.9$ & $31.5 \pm 1.2$ & $19.8 \pm 1.3$ & $38 \pm 1$ \\
\hline
\end{tabular}

Table 2. Study Results.

\begin{tabular}{clccccc}
\hline \multirow{2}{*}{$\begin{array}{c}\text { Microorganism, place of } \\
\text { washing }\end{array}$} & \multicolumn{5}{c}{ Inhibition zones, mm } \\
\cline { 2 - 6 } & TML & TMS & FLF & CFF & EFC \\
\hline 1 & $\begin{array}{l}\text { Streptococcus suis } \\
\text { (synovial fluid) }\end{array}$ & $11.2 \pm 1.4$ & 0 & $23.2 \pm 1.4$ & $22.7 \pm 1.3$ & $21.5 \pm 1.9$ \\
\hline 2 & E.coli (exudate, lungs) & $11.7 \pm 1.8$ & $17.8 \pm 1.6$ & $28.7 \pm 2.2$ & $26 \pm 2$ & $36.3 \pm 2.5$ \\
\hline 3 & $\begin{array}{l}\text { Proteus mirabilis } \\
\text { (exudate, lungs) }\end{array}$ & 0 & 0 & $25 \pm 1.5$ & $29.2 \pm 1.7$ & $12 \pm 0.5$ \\
\hline 4 & E. c oli (liver) & $18.7 \pm 0.8$ & $15 \pm 0.7$ & $20.7 \pm 1$ & $23.8 \pm 1.7$ & $30.8 \pm 1.1$ \\
\hline 5 & Myroides phaeus (liver) & $16 \pm 0.5$ & $19 \pm 0.5$ & $19.2 \pm 0.6$ & $17 \pm 0.5$ & $13.8 \pm 0.8$ \\
\hline 6 & $\begin{array}{l}\text { Streptococcus canis } \\
\text { (pericardium) }\end{array}$ & $18.8 \pm 1.2$ & $21 \pm 1.3$ & $26.2 \pm 1.2$ & $33.2 \pm 1.1$ & $20.5 \pm 1.1$ \\
\hline 7 & E. col, (pericardium) & $15 \pm 0.9$ & $18.2 \pm 0.8$ & $20.8 \pm 1.2$ & $23.8 \pm 1.3$ & $30.8 \pm 1.2$ \\
\hline 8 & E. c oli (exudate, lungs) & $10.7 \pm 0.4$ & $14 \pm 0.5$ & $22.2 \pm 0.6$ & $24.9 \pm 1$ & $19.2 \pm 0.6$ \\
\hline 9 & $\begin{array}{l}\text { Proteus mirabilis } \\
\text { (exudate, lungs) }\end{array}$ & 0 & 0 & $15.2 \pm 0.6$ & $21 \pm 0.7$ & $30.7 \pm 0.7$ \\
\hline 10 & $\begin{array}{l}\text { Klebsiella pneumonia } \\
\text { (exudate, lungs) }\end{array}$ & $11 \pm 0.7$ & $18.2 \pm 1.5$ & $20.3 \pm 1.3$ & $24.8 \pm 1.3$ & $30.2 \pm 1.5$ \\
\hline 11 & $\begin{array}{l}\text { Providencia rettgeri } \\
\text { (exudate, lungs) }\end{array}$ & 0 & 0 & $9.8 \pm 0.6$ & 0 & 0 \\
\hline 12 & E. c oli (pericardium) & $14 \pm 1.1$ & $12.8 \pm 0.6$ & $20.3 \pm 1.3$ & $22.8 \pm 0.6$ & $21.8 \pm 1.2$ \\
\hline & & & & & & \\
\hline
\end{tabular}




\begin{tabular}{|c|c|c|c|c|c|c|}
\hline \multirow{2}{*}{\multicolumn{2}{|c|}{$\begin{array}{c}\text { Microorganism, place of } \\
\text { washing }\end{array}$}} & \multicolumn{5}{|c|}{ Inhibition zones, mm } \\
\hline & & \multirow{2}{*}{$\begin{array}{c}\text { TML } \\
0\end{array}$} & \multirow{2}{*}{$\begin{array}{c}\text { TMS } \\
0\end{array}$} & \multirow{2}{*}{$\begin{array}{c}\text { FLF } \\
13.8 \pm 0.6\end{array}$} & \multirow{2}{*}{$\begin{array}{c}\text { CFF } \\
19 \pm 0.5\end{array}$} & \multirow{2}{*}{$\begin{array}{c}\text { EFC } \\
19.3 \pm 1.1\end{array}$} \\
\hline 13 & $\begin{array}{l}\text { Myroides odoratus } \\
\text { (pericardium) }\end{array}$ & & & & & \\
\hline 14 & E.coli, (exudate, lungs) & $11.5 \pm 1$ & $14.2 \pm 1$ & $21.4 \pm 0.7$ & $24.8 \pm 1.2$ & $20.5 \pm 1.1$ \\
\hline 15 & $\begin{array}{l}\text { Proteus mirabilis } \\
\text { (exudate, lungs) }\end{array}$ & 0 & 0 & $25 \pm 1.1$ & $25.3 \pm 1.3$ & $29 \pm 1.1$ \\
\hline 16 & $\begin{array}{l}\text { Vagococcus fluvialis } \\
\text { (exudate, lungs) }\end{array}$ & 0 & 0 & $23.2 \pm 1.2$ & $15.3 \pm 1.2$ & $13.8 \pm 0.6$ \\
\hline 17 & E. c oli (exudate, lungs) & $14.8 \pm 1$ & $15.8 \pm 1$ & $17.8 \pm 0.6$ & $18.2 \pm 0.8$ & $31 \pm 1.1$ \\
\hline 18 & $\begin{array}{l}\text { Proteus mirabilis } \\
\text { (exudate, lungs) }\end{array}$ & 0 & 0 & $21.2 \pm 0.8$ & $29.3 \pm 1.1$ & $12.9 \pm 0.9$ \\
\hline 19 & $\begin{array}{l}\text { Aeromonas hydrophila } \\
\text { (exudate, lungs) }\end{array}$ & 0 & 0 & $25 \pm 0.9$ & $17.8 \pm 1$ & $10.9 \pm 0.9$ \\
\hline 20 & E. c oli (synovial fluid) & $11.2 \pm 0.6$ & $12.8 \pm 0.8$ & $18.2 \pm 0.6$ & $22.2 \pm 1.1$ & $28 \pm 1.3$ \\
\hline 21 & $\begin{array}{l}\text { Morganella morganii } \\
\text { (synovial fluid) }\end{array}$ & 0 & 0 & $16 \pm 0.9$ & $26 \pm 1.1$ & $9 \pm 0.5$ \\
\hline 22 & E. c oli (exudate, lungs) & $12 \pm 0.7$ & $15.2 \pm 0.6$ & $21 \pm 1.5$ & $21.2 \pm 1$ & $26 \pm 0.9$ \\
\hline 23 & $\begin{array}{l}\text { Proteus mirabilis } \\
\text { (exudate, lungs) }\end{array}$ & 0 & 0 & $27.8 \pm 0.8$ & $32 \pm 0.9$ & $9 \pm 0.5$ \\
\hline 24 & $\begin{array}{l}\text { Providencia rettgeri } \\
\text { (exudate, lungs) }\end{array}$ & 0 & 0 & $19 \pm 0.9$ & $27 \pm 0.9$ & $30,2 \pm 1.3$ \\
\hline 25 & $\begin{array}{l}\text { Aeromonas hydrophila } \\
\text { (exudate, lungs) }\end{array}$ & 0 & 0 & $23 \pm 0.9$ & $20 \pm 0.9$ & $11.8 \pm 0.8$ \\
\hline & $\begin{array}{l}\text { Control E. coli ATCC } \\
25922\end{array}$ & $29.2 \pm 1.2$ & $30.5 \pm 1.5$ & $30.2 \pm 1.5$ & $39.8 \pm 1.3$ & $35.2 \pm 1.1$ \\
\hline
\end{tabular}

\section{Discussion}

Identification was considered valid if the service assigned the analyzed results a score of "Good identification" and "Excellent identification". The standard deviation of the parallel growth inhibition values did not exceed 2.0. Interpretation of the obtained sizes of the growth inhibition zones is difficult: the standard [8] does not include the full range of the studied materials. It shall also be noted that standards [10] and [11] similarly do not contain information for distribution of all obtained isolates into groups (sensitive, moderately sensitive, resistant) to a particular substance used in the experiment with antimicrobial properties. To interpret the results that are not unambiguously described in the current standards for antimicrobial susceptibility (MUK RF, CLSI, EUCAST), it is possible to use the explanations to the standard [11] described in appendix [12].

The data obtained by us were interpreted according to the fact of the complete absence of the growth inhibition zone around the disc with the antimicrobial drug. 18 out of 25 isolated strains showed complete resistance (no zone of inhibition) to at least one of the antimicrobial substances. Where, strains 3, 9, 11, 13, 15, 16, 19, 21, 24, 25 are multiresistant (resistance to three or more antimicrobial substances of different classes), which is $40 \%$ of the total number of isolates studied.

\section{Conclusion}

As a result of the work carried out, we have identified multi-resistant strains of microorganisms. There are no cases of complete resistance to all tested antimicrobial substances. The highest efficiency in relation to the studied cultures was shown by 
enrofloxacin, ceftiofur, levofloxacin (1 strain with full resistance out of 25 studied), azitronite ( 2 out of 25). No strains have been identified that are fully resistant to florfenicol.

\section{References}

1. M. Tumbarello, E. M. Trecarichi, B. Fiori, A. R. Losito, T. D'Inzeo, L. Campana, A. Ruggeri, M.E. Di, E. Liberto, G. Fadda, R. Cauda, T. Spanub, Antimicrob Agents Chemother, 56 (2012)

2. M. Bassetti, E. Righi, A. Carnelutti, E. Graziano, A. Russo, Expert Rev Anti Infect Ther, 16 (2018)

3. H. Shao-hua, Y. Shu-xing, Q.Hai, J. Tao, Z. Ya-jun, W. Ming-xi, J. Zhejiang Univ Sci B, 17 (2016)

4. Y. Sun, Sh. Wen, L. Zhao, Q. Xia, Y. Pan, H. Liu, Ch. Wei, H. Chen, J. Ge, H. Wang, BMC Veterinary Research, 16, 176 (2020)

5. R. Sally, S. M. Kwong, F. Neville, O J. Slade, Clin Microbiol Rev., 31, 4 (2018)

6. Z. YU. Hapcev, V. A. Agol'cov, R. V. Radionov, G. A. ZHukova, Nauchnaya zhizn', $1(2020)$

7. Z. YU. Hapcev Mezhvuzovskij sbornik nauchnyh trudov II Vcerossijskoj konferencii s mezhdunarodnym uchastiem (Saratov, 2019)

8. Perfomance Standards for Antimicrobial Disk and Dilution Susceptibility Tests for Bacteria isolated from animals (Clinical and laboratory standards institute, Wayne, USA, 2003)

9. G. F. Lakin, Biometriya (Moskva, 1990)

10. Pogranichnye znacheniya MPK i diametrov zon podavleniya rosta dlya interpretacii rezul'tatov opredeleniya chuvstvitel'nosti (EUCAST, 2021)

11. Opredelenie chuvstvitel'nosti mikroorganizmov $\mathrm{k}$ antibakterial'nym preparatam (Federal'nyj centr gossanepidnadzora Minzdrava Rossii, 2004)

12. Antimicrobial susceptibility tests on groups of organisms or agents for which there are no EUCAST breakpoints (EUCAST, 2016) 